Document downloaded from:

http://hdl.handle.net/10251/176196

This paper must be cited as:

Pérez Pascual, MA.; Bruno, JS.; Almenar Terre, V.; Valls Coquillat, J. (2020). A Computational Efficient Nyquist Shaping Approach for Short-Reach Optical

Communications. Journal of Lightwave Technology (Online). 38(7):1651-1658. https://doi.org/10.1109/JLT.2019.2961506

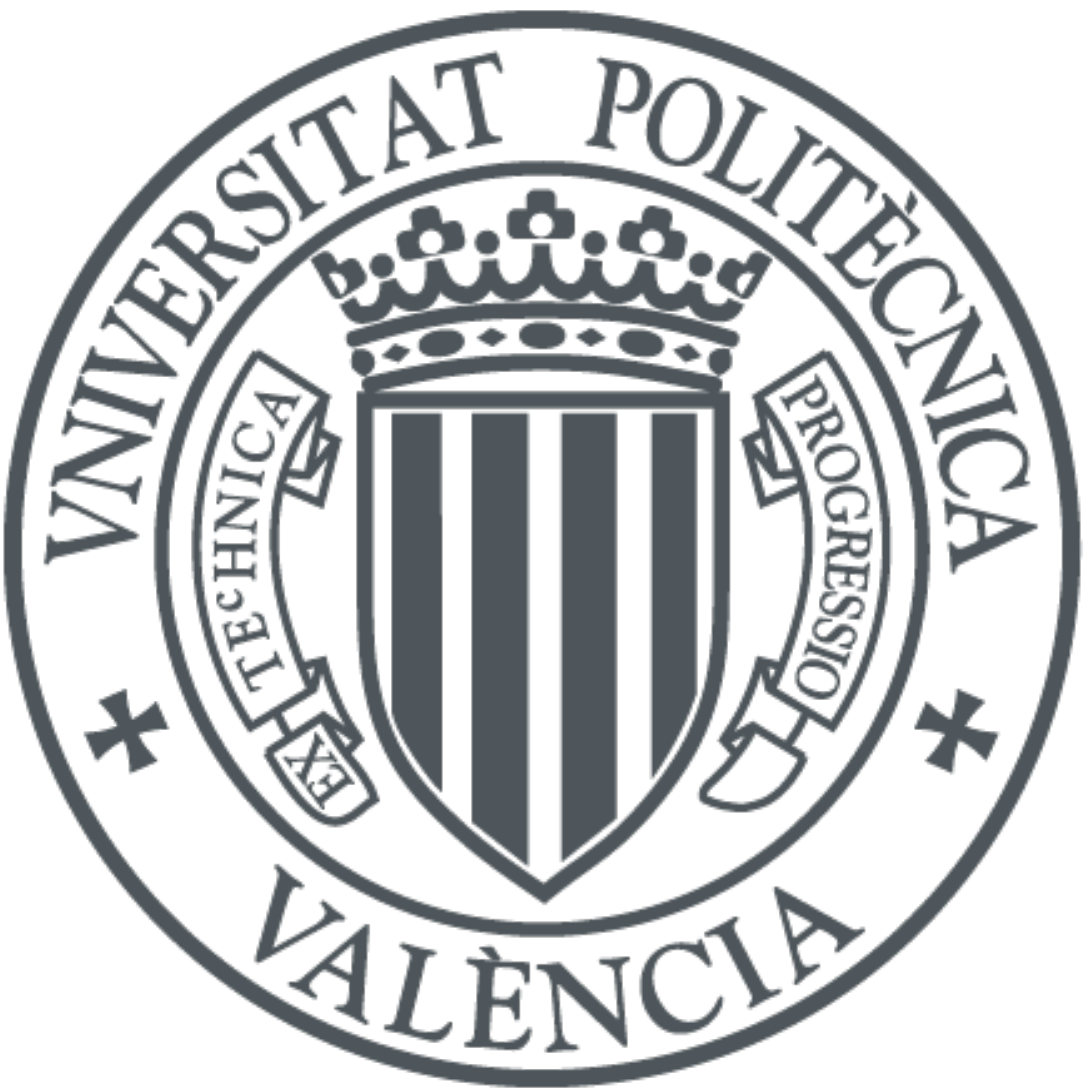

The final publication is available at

https://doi.org/10.1109/JLT.2019.2961506

Copyright Institute of Electrical and Electronics Engineers

Additional Information 


\title{
A Computational Efficient Nyquist Shaping Approach for Short-Reach Optical Communications
}

\author{
Asun Pérez-Pascual (D), Julián S. Bruno (D), Vicenç Almenar (D), and Javier Valls (D)
}

\begin{abstract}
Recently, Half-Cycle Nyquist Subcarrier Modulation (HC-SCM) was proposed to achieve high spectral efficiency in intensity-modulator direct-detection optical links. This paper shows that the HC-SCM scheme has a high computational load and proposes the rational Oversampled Subcarrier Modulation (OVS-SCM) as a computational efficient alternative that, furthermore, improves the spectral efficiency. The presented experimental results show that our 256-QAM proposal allows to transmit below the hard-decision forward error correction, with a throughput of $17.8 \mathrm{~Gb} / \mathrm{s}$ in a $2.5 \mathrm{GHz}$ bandwidth, and a spectral efficiency of $7.2 \mathrm{~b} / \mathrm{s} / \mathrm{Hz}$, through 20 km of single-mode optical fiber.
\end{abstract}

Index Terms-DSP, Fiber optic, FPGA, IM/DD

\section{INTRODUCTION}

$\mathbf{T}$ HE volume of data traffic in telecommunication networks is increasing year after year. As a result of this demand, optical links in data centers and interconnect applications will require to operate at higher data rates. Not only is more transmission capacity requested, but also a reduction in capital and operating expenses is necessary. In order to provide this increasing user rates at limited costs of equipment, short reach optical fiber systems with intensity modulated with direct-detection systems (IM/DD) over standard single-mode fiber (SSMF) are a practical solution.

On the other hand, electronic manufactures have anticipated this need and have developed transceivers, converters and programmable devices that support high throughput and can be integrated in next-generation optics [1]. Within these devices, digital signal processing techniques [2] can be used to improve the receiver performance via several solutions: the compensation of inter-symbol interference thanks to the use of equalizers; compensation of transmission impairments, such as phase and amplitude distortion, which reduces the cost of the optic devices; and the inclusion of Forward Error Correction (FEC) techniques to increase optical power budget at a relative lower cost [3].

Next-generation short reach optical systems are being researched with the aim of improving the spectrum efficiency. A large proportion of the works found in the literature related to this topic makes use of Orthogonal Frequency-Division Multiplexing (OFDM) [4], [5], [6]. In contrast to OFDM, Nyquist signaling [7], [8] offers an interesting alternative that is deeply developed throughout this work.

Single Cycle Subcarrier modulation (SCM) was first introduced in 2009 at [9] as an alternative to multilevel optical phase and amplitude links. The goal of this proposal was to modulate data on a RF-subcarrier whose frequency is equal to the symbol rate. In this way, the complexity of the system is moved towards the electrical domain, reducing the cost of the whole system. In [9] this approach was presented and experimentally demonstrated implementing a 16-Phase Shift Keying (PSK) and a 16-Quadrature Amplitude Modulation (QAM) optical link, a throughput of $10 \mathrm{~Gb} / \mathrm{s}$ was achieved.

In [10] another approach based on SCM was presented. In this case, the data waveform was shaped using a Nyquist pulse in order to limit its spectrum. A raised cosine pulse with a roll-off factor of zero was employed, occuping a bandwidth equal to the symbol rate. This proposal used a subcarrier frequency equal to half of the symbol rate, and was named as Half-Cycle (HC). One of the main achievements of this experiment is that the required digital to analog converter (DAC) sampling rate is decreased compared to [9]. Bit rates of 14,28 and $56 \mathrm{~Gb} / \mathrm{s}$ were measured with a 16-QAM transmitter and its corresponding receiver, making both use of digital signal processing (DSP) algorithms, to demonstrate the viability of this proposal.

Based on the previous approach, the work presented in [7] improves the spectral efficiency implementing a $32.5 \mathrm{~Gb} / \mathrm{s}$ 64-QAM Half-Cycle subcarrier modulator (HC-SCM) and a $38 \mathrm{~Gb} / \mathrm{s}$ 128-QAM HC-SCM. The evaluation carried out in that work concluded that the bit error rate was under the hard decision forward error correction (HD-FEC) threshold of $3.8 \cdot 10^{-3}$ for $64-\mathrm{QAM}$ and under soft-decision forward error correction (SD-FEC) threshold of $2.4 \cdot 10^{-2}$ for 128-QAM [11].

Finally, in 2015, a 112Gbit/s half-cycle Nyquist 16-QAM SCM system was generated based on dual wavelength schemes [12]. The cited works only present experimental studies, without taking into account the computational load of the algorithms required to achieve these results. In some cases, their complexity is so high that their implementation would be unaffordable.

In this paper we propose a computationally feasible alternative to Half-Cycle subcarrier modulation based on an oversampled Nyquist pulse. We compare the performance of both Half-Cycle and the proposed alternative with experimental measurements in a short-length passive IM/DD optical link using high-order modulation formats such as 64 , 128 and 256-QAM

The outline of the paper is the following: in section II the Half-Cycle Subcarrier modulation (HC-SCM) transmission system is introduced and tested over an experimental setup and its computational load is calculated. Section [III details the rational Oversampling Nyquist Subcarrier modulation, which is tested with the same experimental setup. Section IV] compares the results in terms of throughput, bit error rate (BER), error vector magnitude (EVM) and computational load 
(CL), moreover the BER vs. received optical power is shown. Finally, conclusions are exposed in section $\mathrm{V}$

\section{HALF-CyCle NyQuist Sub-CARRIER Modulation TRASMISSION SYSTEM}

In this section, the Half-Cycle subcarrier modulation approach [10] is described and experimentally evaluated. Figure 1 presents the transmitter scheme: data bits are grouped in M-QAM complex symbols at a symbol rate of $1 / \mathrm{T} \mathrm{Hz}$ and, then, their real and imaginary parts feed the in-phase (I) and quadrature (Q) branches, respectively. Next, after an upsampling by 2, a Nyquist pulse shaping filter (a root-raised cosine digital filter) with a roll-off factor $(\beta)$ is employed to confine the base band (BB) signal spectrum bandwidth (BW) according to Eq. 1 .

$$
B W_{B B}=\frac{1}{2 T} \cdot(1+\beta)
$$

A roll-off factor of $\beta=0$ is used to reduce the occupied bandwidth. The sampling frequency at this point is $F_{s}=2 / T$. After that, each branch is upconverted by a cosine and a sine with a frequency of one quarter of the sampling frequency $f_{c}=F_{s} / 4$, which is equal to $f_{c}=\frac{1 / T}{2}$, that is, half of the symbol rate, and corresponds to a digital frequency of $\omega_{c}=\pi / 2$. The use of a $\beta=0$ and the selected $\omega_{c}$ confines the signal spectrum inside the Nyquist bandwidth (between 0 and $1 / T$ ) and avoids, in this way, any aliasing. Finally, a second-order finite impulse response (FIR) filter pre-emphasizes the spectrum signal to compensate the DAC low-pass frequency response. The DAC generates samples at a rate of $F_{S}$ samples per second.

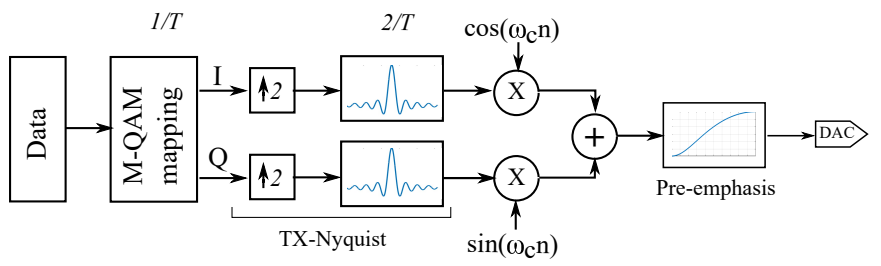

Fig. 1: Half-Cycle Nyquist SCM Transmitter

The receiver block diagram is shown in Fig2, in this case the analog to digital converter (ADC) works at the same sampling frequency as the DAC, that is, two samples per symbol. After downconversion to baseband, the signal is filtered by a matched filter and then downsampled by 2 to reverse the operations done at the transmitter. These symbol samples are equalized and de-mapped to obtain the received bits.

A preamble using QPSK training symbols is transmitted before the data symbol signal to estimate the timing phase and the equalizer coefficients. The former is estimated making use of the square timing recovery algorithm [13], then the obtained fractional delay is employed to adjust the root-raised cosine impulse response filter of the receiver. In this way, not only is this filter used for the matching filter task, but also corrects the time delay. This preamble is also used to calculate the equalizer coefficients by means of the minimum mean square error algorithm, then they are employed to implement a symbol spaced linear feed-forward equalizer, which is applied at the decimator output.

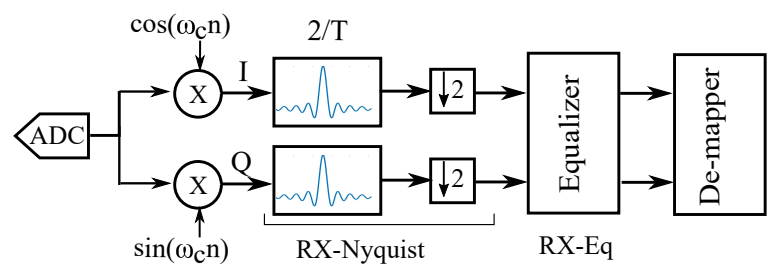

Fig. 2: Half-Cycle Nyquist SCM Receiver

\section{A. Experimental setup}

The HC-SCM solution was tested using an IM/DD optical communication link setup, which is shown in Fig. 3 The samples of the HC-SCM signal were generated in MATLAB and then sent to a field programmable gate array (FPGA), Xilinx FPGA evaluation board VC707, equipped with an Euvis DAC MD657B (12 bit) operating at $5.0 \mathrm{GS} / \mathrm{s}$. After the data converter, the analog signal was amplified before the electro-optical conversion, which was done with a low cost directly modulated laser (DML) operating at $1550 \mathrm{~nm}$ wavelength with a $+4.3 \mathrm{dBm}$ optical output power.

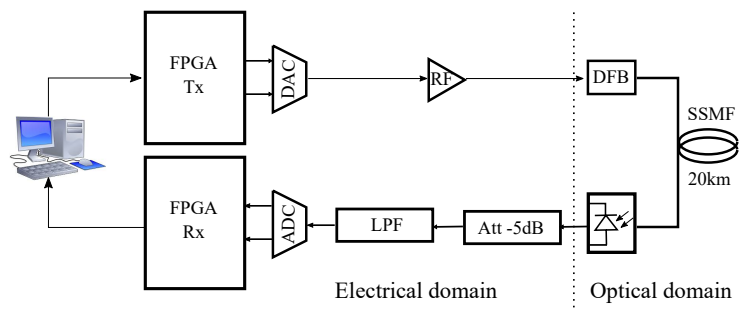

Fig. 3: Experimental Setup Block diagram. The transmitted and received signal are processed offline in Matlab.

This signal was transmitted through $20 \mathrm{~km}$ of SSMF whose attenuation is $0.19 \mathrm{~dB} / \mathrm{km}$. At the fiber output, the signal had a $0.2 \mathrm{dBm}$ optical power and was converted again to the electrical domain using an InGaAs PIN photodiode with a responsivity of $0.94 \mathrm{~A} / \mathrm{W}$ at $1550 \mathrm{~nm}$. The whole electrical to optic and optic to electrical system had a RF bandwidth of $3 \mathrm{GHz}$. Before sampling, the amplitude of the photodetected signal was adjusted with an attenuator to avoid saturation at the ADC. Then, it was filtered using a low-pass filter (LPF) to avoid aliasing and out-of-band noise with the following specifications: a $3 \mathrm{~dB}$ bandwidth of $2.35 \mathrm{GHz}$, a maximum of $1.8 \mathrm{~dB}$ insertion loss and a minimum rejection of $40 \mathrm{~dB}$ from $2.65 \mathrm{GHz}$. Then, the filtered signal was sampled at $5 \mathrm{GS} / \mathrm{s}$ by an E2V ADC EV10AQ190A (10 bits) connected to another FPGA evaluation board. Finally, the captured signal was sent to the computer, via Ethernet, where the received signal was processed offline in Matlab.

\section{B. Experimental results}

Before assessing the performance of the HC-SCM scheme it is necessary to adjust the order of the employed filters. On 
the one hand, the raised cosine filters (both at transmitter and receiver) with $\beta=0$ require a high order to obtain a brick-wall response, which is necessary to avoid aliasing when the signal is upconverted to the mid-band subcarrier $f_{c}=F_{S} / 4$. The length of the raised cosine impulse response $\left(M_{R C}\right)$ is usually measured in number of symbols, which is called the span of the filter. The filter order is obtained multiplying the span by the number of samples per symbol $\left(N_{s p s}, 2\right.$ in this case) and adding 1 for the central coefficient:

$$
M_{R C}=\operatorname{span} \cdot N_{\text {sps }}+1
$$

On the other hand, the order of the equalizer must be chosen to reduce interferences from both the channel and the residual aliasing due to non-ideal filtering.

The experimental setup described in the previous section was employed to evaluate the performance of the HC-SCM for different filter order values. The EVM of the received symbols was measured to adjust the span length and the equalizer order. Fig. 4 shows how as the span grows the EVM decreases, that is, the performance is improved, but this gain stops for a span of 350 symbols. In the same figure, it can also be seen that an equalizer order of 200 gives the lower EVM. Once these parameters were fixed, the BER performance of the HC-SCM scheme was measured. Table I] shows the BER and the EVM for three different modulation orders: 64, 128 and 256-QAM after $20 \mathrm{~km}$. It can be observed that only 64-QAM can achieve a BER lower than the HD-FEC threshold of $3.8 \cdot 10^{-3}$ [11].

Figure 5a shows the 64-QAM signal spectrum measured with a spectrum analyzer after the DAC. As the DAC sampling frequency is $5 \mathrm{GHz}$, the band of interest where the HC-SCM signal falls is between 0 and $2.5 \mathrm{GHz}$, next to it, without any band-guard, the DAC generates an attenuated replica in the second Nyquist zone. This one is mostly cancelled before the ADC thanks to the antialiasing LPF, whose signal spectrum output is shown in Fig. 5b. It can be seen how the filter cannot cancel completely the replica and this will alias the signal bandwidth after the sampling. Finally, Fig. 5c represents the constellation diagram of the received symbols.

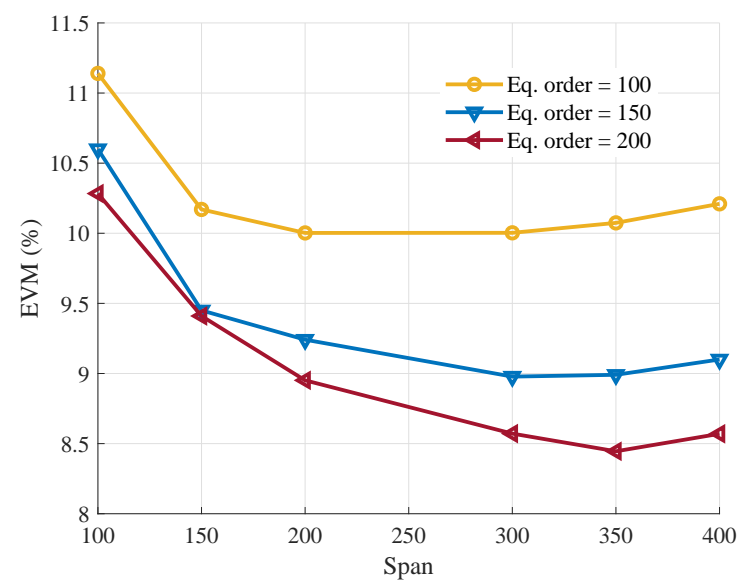

Fig. 4: 64-QAM HC-SCM transmissor: EVM vs Nyquist pulse span for different Equalizer orders (100, 150 and 200)
TABLE I: HC-SCM THROUGHPUT, BER AND EVM EXPERIMENTAL RESULTS (Nyquist span=350, Equalizer order $=200$ )

\begin{tabular}{cccc}
\hline \hline Modulation & $\begin{array}{c}\text { Throughput } \\
(\mathrm{G} b / \mathrm{s})\end{array}$ & BER & $\begin{array}{c}\text { EVM } \\
\%\end{array}$ \\
\hline 64-QAM & 15 & $2.9 \cdot 10^{-3}$ & 8.4 \\
128-QAM & 17.5 & $1.58 \cdot 10^{-2}$ & 8.5 \\
256-QAM & 20 & $4 \cdot 10^{-2}$ & 8.6 \\
\hline \hline
\end{tabular}

\section{Computational load}

The computational load, measured as the number of multiplications per second, is estimated for each module and for the whole HC-SCM system. The Nyquist pulse filters of Fig. 1 and Fig. 2 can be implemented with a polyphase structure [14], which is a well-known computationally efficient approach to implement multirate filters. The interpolation or decimation by $\mathrm{R}$ of an $\mathrm{N}$-order filter is implemented using a polyphase filter as a bank of $\mathrm{R} N$ /R-order subfilters. In such a case, its $C L$ is reduced by a factor $R$ with respect to the direct implementation of those filters. This reduction is achieved because although the same number of multiplications is computed in both cases, they are done at the high sampling rate $\left(f_{S H}\right)$ in the direct implementation, whereas at the low sampling rate $\left(f_{S L}=f_{S H} / R\right)$ in the polyphase one.

Below is detailed the calculus of the CL.

1) The pulse shaping Nyquist filter (TX-Nyquist) used in the transmitter has (span.2 +1 ) coefficients and its output operating frequency is $5 \mathrm{GHz}$, as can be deduced from Fig. 1. As it can be implemented as an interpolator by two polyphase filter, the number of multiplications per second is reduced by half. Its CL is:

$$
C L_{T X-N y q .}=\left(\frac{\operatorname{span} \cdot 2+1}{2}\right) \cdot 5 \mathrm{GMult} / \mathrm{s} .
$$

2) The mixers in both, the transmitter and the receiver, do not introduce any multiplication because their subcarrier frequency is $F_{s} / 4$ (i.e. $\cos (2 \pi n / 4)=(1,0,-1,0)$ and $\sin (2 \pi n / 4)=(0,1,0,-1)$ for $n=0 \ldots 3)$.

3) The CL of the second-order pre-emphasis filter is estimated as:

$$
C L_{\text {pre }}=2 \cdot 5 \text { GMult } / \mathrm{s} .
$$

4) The receiver Nyquist pulse (RX-Nyquist) is a decimator by two polyphase filter and has also $(\operatorname{span} \cdot 2+1)$ coefficients. Its $\mathrm{CL}$ is:

$$
C L_{R X-N y q .}=\left(\frac{\operatorname{span} \cdot 2+1}{2}\right) \cdot 5 \text { GMult } / \mathrm{s} .
$$

5) Finally, the computational load of the $M$-order equalizer is:

$$
C L_{R X-E q}=M \cdot 2.5 \mathrm{GMult} / \mathrm{s} .
$$

Tables III and III present the CL for both parts, the transmitter and the receiver of the HC-SCM system. The Nyquist pulse span is fixed to 350 samples, and the equalizer order to 200. As expected, the receiver has a CL higher than the one of the transmitter due to the equalizer. The CL of the whole system is $8020 \mathrm{GMult} / \mathrm{s}$. This calculation does not 


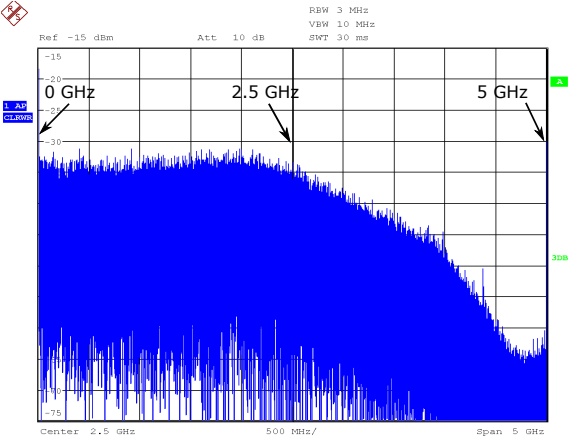

(a)

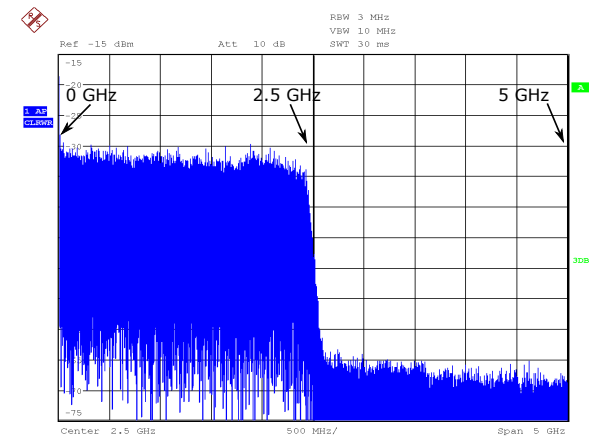

(b)

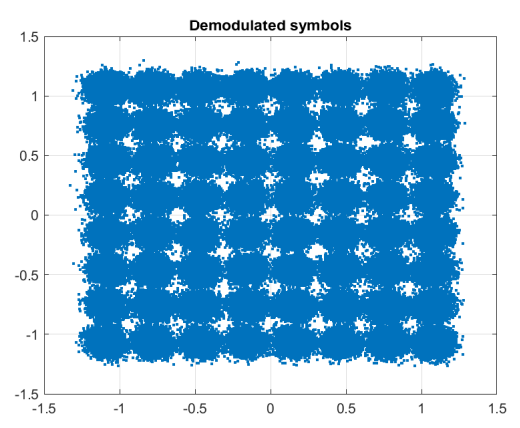

(c)

Fig. 5: 64-QAM HC-SCM Signal Spectrum after DAC (a), after anti-alising filter (b) and received constellation (c)

include the CL of timing phase estimation as this is done once during the preamble processing and its cost is much lower than the rest of the receiver filtering. Additionally, our proposal also requires timing phase estimation, whose complexity is similar to $\mathrm{HC}-\mathrm{SCM}$, so the comparison is not affected.

\section{OVERSAMPLED SCM TRANSMISSION SYSTEM}

The experimental results presented in section III show that although the HC-SCM scheme is an interesting solution to achieve high spectral efficiency in IM-DD optical links, it comes at the expense of a very high computational cost. The main reason for this complexity is the use of high-order raised cosine digital filters, which are needed because the QAM signal spectrum must be tightly confined to avoid aliasing. This section is devoted to present an alternative approach to HC-SCM that requires a much lower $\mathrm{CL}$ and, at the same time, improves the spectral efficiency.

The only way to reduce the order of the filters is to have a filter frequency response with a transition bandwidth, instead of using a brick wall frequency response as in HC-SCM. This can be accomplished using a roll-off factor higher than 0 , but, at the same time, low enough to avoid wasting the transmission spectrum. With this condition in mind, $\beta=0.1$ was chosen, thus a $10 \%$ of the Nyquist band spectrum is dedicated to the transition band. As a consequence, the use of a $\beta>0$ requires the use of more than 2 samples per symbol, for $\beta=0.1$ it is necessary to sample at a rate of 2.2 samples per symbol or higher. As this solution makes use of a higher sample rate than HC-SCM, we have called it oversampled SCM (OVS-SCM).

It is possible to generate a waveform with a non-integer sampling rate combining an interpolation and a decimation stages, both with integer factors. The rational number with lower values that is nearer to 2.2 is $9 / 4$, which gives a rate of 2.25 samples per symbol. An efficient way to implement this non-integer oversampling ratio is to divide it in two sections: the first one is a pulse shaping filter composed of an upsampling by 3 followed by a Nyquist root-raised cosine digital filter, and the second one is composed of an upsampling by 3 , a low-pass filter and a downsampling by 4 , as is shown in Fig. 6 As in the HC-SCM implementation, a pre-emphasis filter is also used to compensate for the DAC low pass frequency response.
TABLE II: HC-SCM TX COMPUTATIONAL LOAD

\begin{tabular}{ccccc}
\hline $\begin{array}{c}\text { TX-Nyq. } \\
\text { span }\end{array}$ & $\begin{array}{c}C L_{T X-N y q .} \\
(\text { Gmult } / s)\end{array}$ & $\begin{array}{c}\text { Pre-emph } \\
\text { order }\end{array}$ & $\begin{array}{c}C L_{p r e} \\
(\text { Gmult } / s)\end{array}$ & $\begin{array}{c}\text { Total CL } \\
(\text { Gmult } / s)\end{array}$ \\
\hline 350 & 1752.5 & 2 & 10 & 3515 \\
\hline \hline
\end{tabular}

TABLE III: HC-SCM RX COMPUTATIONAL LOAD

\begin{tabular}{ccccc}
\hline \hline $\begin{array}{c}\text { RX-Nyq. } \\
\text { span }\end{array}$ & $\begin{array}{c}C L_{R X-N y q .} \\
(\text { Gmult } / s)\end{array}$ & $\begin{array}{c}\text { Equalizer } \\
\text { order }\end{array}$ & $\begin{array}{c}C L_{R X-E q} \\
(\text { Gmult } / s)\end{array}$ & $\begin{array}{c}\text { Total CL } \\
(\text { Gmult } / s)\end{array}$ \\
\hline 350 & 1752.5 & 200 & 500 & 4505 \\
\hline \hline
\end{tabular}

With this approach it is still possible to upconvert the baseband signal to the same subcarrier frequency of $F_{S} / 4 \mathrm{~Hz}$ as in HC-SCM. This is equivalent to the digital frequency $\omega_{c}=\pi / 2$, so, the low-cost upconversion is maintained. But, as the sampling frequency of the DAC does not change, the use of a higher number of samples per symbol gives a lower symbol rate: $1 / T=F_{S} / 2.25$. Later, when we will discuss the experimental results, it will be seen that, in spite of this loss, the overall spectral efficiency is improved.

The block diagram of the receiver is presented in Fig. 7 It can be seen how after downconversion to baseband, the signal is upsampled by 4 , filtered and then downsampled by 3 to reverse the operations done at the transmitter. Finally, a matched filter using a Nyquist root-raised cosine impulse response followed by a downsampling by 3 gives an output sampled at the symbol rate. These symbol samples are equalized and passed to the de-mapper to obtain the received bits. Time synchronization is solved in the same way as commented previously.

Once the structures of the transmitter and receiver are established, next phase is to design the filters. As we did with HC-SCM, the span of the Nyquist filters in the OVS-SCM scheme and the equalizer order will be adjusted by means of measurements in next subsection. The low pass filters used in the non-integer rate conversion (labelled as TX-9/4 LPF and RX-9/4 LPF in Fig. 6 and Fig. 7) respectively) were designed following these guidelines: a pass band ripple of less than $0.1 \mathrm{~dB}$, an attenuation higher than $48 \mathrm{~dB}$ and a nominal cut frequency of $\omega_{c u t}=\pi / 4$. A Parks-McClellan algorithm was employed, giving as a result a filter length of 20 coefficients. 


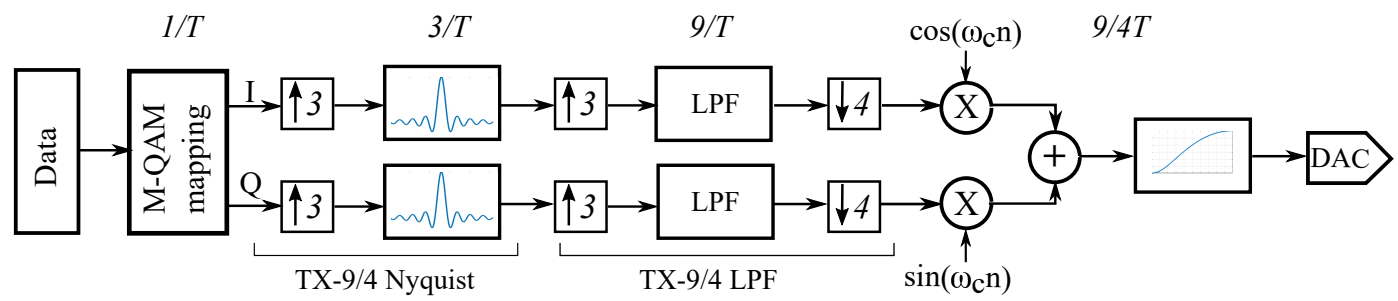

Fig. 6: 9/4 Oversampled SCM Transmitter

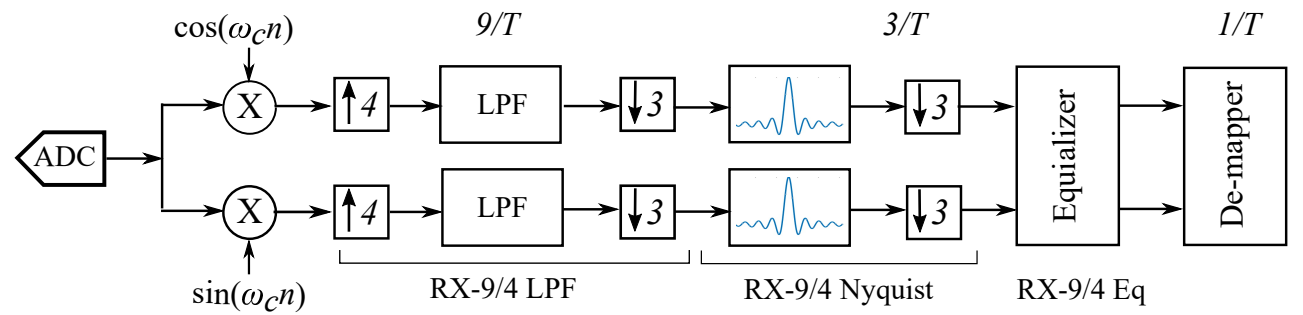

Fig. 7: 9/4 Oversampled SCM Receiver

\section{A. Experimental results}

The OVS-SCM scheme was evaluated using the experimental setup in order to search the best span and equalizer order, similarly to what was done in section II-B

The measured EVM for each experiment is shown in Figure 8 It can be seen that when span lengths are increased the system works better until it reaches a point where the performance does not improve any more. From this point, the obtained EVM is equivalent to the one given by an untruncated raised cosine pulse shaping filter. At the same time, the results also demonstrate that an equalizer order higher than 40 would not meaningfully improve the performance. Using these values the OVS-SCM system was evaluated using the experimental setup explained in II-A over a $20 \mathrm{~km}$ SSMF link.

Table IV] presents the BER and the EVM for several QAM modulation orders. It can be seen that in all the cases the system reaches BER lower than the threshold established for using HD-FEC $\left(3.8 \cdot 10^{-3}\right)$. Moreover, these values were obtained using an equalization order of 40 . Compared with the $15 \mathrm{~Gb} / \mathrm{s}$ data rate of HC-SCM presented in Table I, the OVS-SCM scheme can achieve a higher data rate $(17.8 \mathrm{~Gb} / \mathrm{s})$ using the same bandwidth of $2.5 \mathrm{GHz}$. It offers a higher spectral efficiency, although the symbol rate is lower, since it can employ a higher modulation order. This is due to the fact that the received signal does not suffer the aliasing from the adjacent band thanks to the guard band.

The last remark can be validated looking at Fig. 9a and Fig. $9 \mathrm{~b}$ where it is shown the spectrum of the 256-QAM signal after the DAC and after the analog antialiasing filter, respectively. In this case, it can be seen how, thanks to the guard band (given by the use of $\beta=0.1$ ), the signal spectrum after the antialiasing filter does not contains components from the adjacent band. Additionally, Fig. 9c illustrates the constellation diagram of the received symbols for a 256-QAM signal.

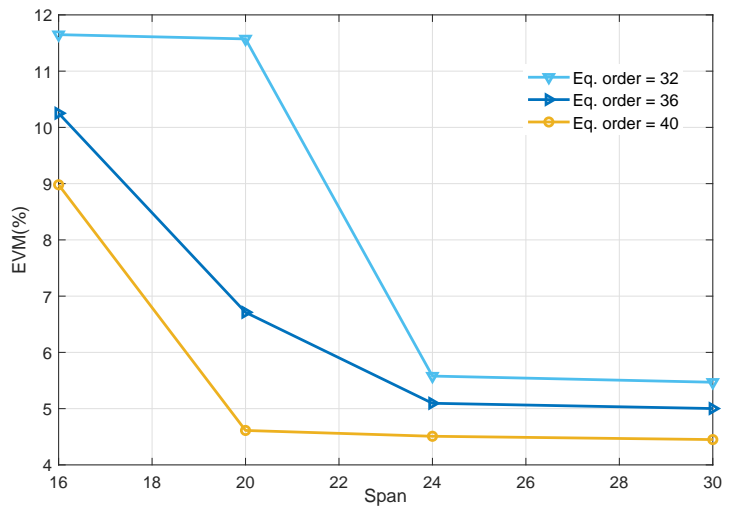

Fig. 8: 9/4 OVS-SCM transmissor: EVM versus Nyquist pulse span for different Equalizer orders (32, 36 and 40)

TABLE IV: 9/4 OVERSAMPLED SCM THROUGHPUT, BER AND EVM RESULTS (Nyquist span=20, Equalizer order $=40$ )

\begin{tabular}{cccc}
\hline \hline Modulation & $\begin{array}{c}\text { Throughput } \\
(G b / s)\end{array}$ & BER & $\begin{array}{c}\text { EVM } \\
\%\end{array}$ \\
\hline 64-QAM & 13.3 & $3 \cdot 10^{-6}$ & 4.6 \\
128-QAM & 15.6 & $6.5 \cdot 10^{-5}$ & 4.5 \\
256-QAM & 17.8 & $2.3 \cdot 10^{-3}$ & 4.5 \\
\hline \hline
\end{tabular}

\section{B. Computational load}

The computational load is estimated for each module and for the whole 9/4 OVS-SCM system.

On the grounds of clarity, the calculus are detailed below.

1) The pulse shaping Nyquist filter (TX-9/4 Nyquist) used in the transmitter has $(\operatorname{span} \cdot 3+1)$ coefficients and its output operating frequency is $(5 \cdot 4 / 3) \mathrm{GHz}$, as can be deduced from Fig. 6 It is implemented as an interpolator by 3 polyphase filter, so the number of multiplications 


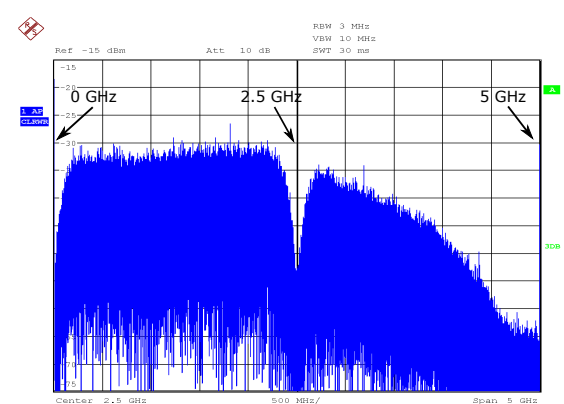

(a)

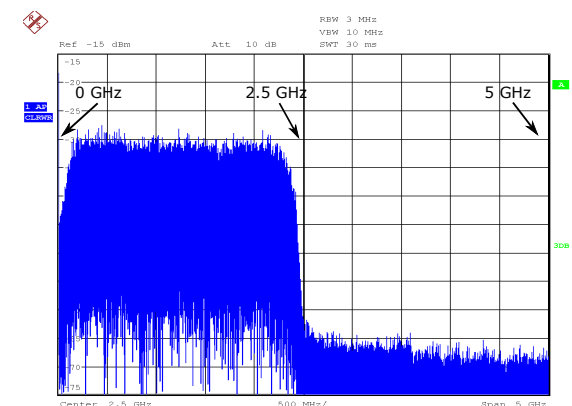

(b)

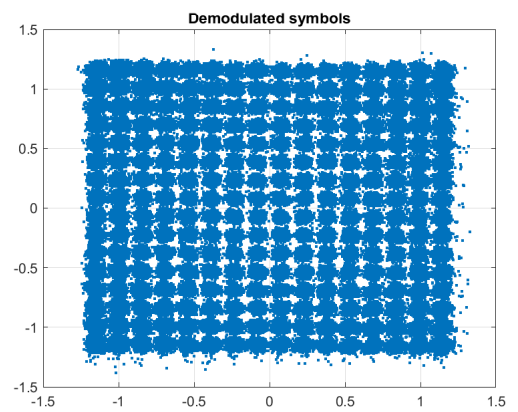

(c)

Fig. 9: 256-QAM OVS-SCM Signal Spectrum after DAC (a), after anti-aliasing filter (b) and constellation (c)

per second is reduced by a factor 3 . Its CL is

$$
C L_{T X-9 / 4 N y q .}=\frac{\operatorname{span} \cdot 3+1}{3} \cdot 5 \cdot \frac{4}{3} \text { GMult } / \mathrm{s} .
$$

2) The N-order TX-9/4 LPF is a rational sampling rate by $3 / 4$ polyphase filter, whose functional scheme is shown in Fig. 10a. It is composed by three $N / 3$-order subfilters followed by a multiplexer that chooses one in four samples. The input of the filter works at a frequency of $3 / T$, and the output is obtained at $9 / 4 T$. In this way the sample rate is changed by $3 / 4$. Its computational load is computed as:

$$
C L_{T X-9 / 4 L P F}=\frac{N}{3} \cdot 5 \mathrm{GMult} / \mathrm{s} .
$$

3) $\mathrm{As}$ in the HC-SCM system, the CL of the second-order pre-emphasis filter is the same as the one in Eq. 4. and the implementation of the mixers does not require multiplications.

4) The receiver RX-9/4 LPF of Fig. 7 is an $N$-order rational sampling rate by $4 / 3$ polyphase filter, whose functional structure is shown in Fig. 10b. It is composed by four $N / 4$-order filters followed by a multiplexer that chooses one in three samples. The input of the filter works at $9 / 4 T$ and the output is obtained at $3 / T$ to change the sample rate by $4 / 3$. Its CL is estimated in Eq. 9 .

$$
C L_{R X-9 / 4 L P F}=\frac{N}{4} \cdot 5 \cdot \frac{4}{3} \mathrm{GMult} / \mathrm{s}
$$

5) The receiver Nyquist filter (RX-9/4 Nyquist) is a decimator by 3 polyphase filter that also has $(\operatorname{span} \cdot 3+1)$ coefficients, where all the coefficients have to be multiplied at the output (low) frequency (i.e. 5 . 4/9 GHz). Its CL is computed using Eq. 10.

$$
C L_{R X-9 / 4 N y q .}=(\operatorname{span} \cdot 3+1) \cdot 5 \cdot \frac{4}{9} \text { GMult } / \mathrm{s}
$$

6) Finally, the $M$-order equalizer works at $(5 \cdot 4 / 9) \mathrm{GHz}$. Its CL is shown in Eq. 11 .

$$
C L_{R X-E q}=M \cdot 5 \cdot \frac{4}{9} G M u l t / s
$$

Tables V and VI present the CL for both parts, the transmitter and the receiver of the 9/4 OVS-SCM system. The Nyquist pulse span is fixed at 20 samples, the order of the $9 / 4$
LPF filters is 20 and the equalizer order is 40 . As in the case of HC-SCM system, the receiver has a higher CL than the one of the transmitter due to the equalizer. The CL of the whole system is $864 \mathrm{GMult} / \mathrm{s}$.

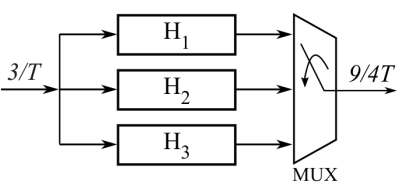

(a)

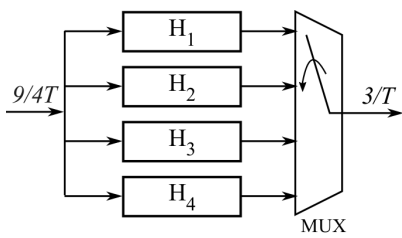

(b)
Fig. 10: Functional schemes of rational sampling rate polyphase filters by $3 / 4$ for transmitter (a) and by $4 / 3$ for receiver (b)

\section{RESULTS COMPARISON}

Results in terms of throughput, BER and EVM for the HC-SCM were presented in Table I] From this table it can be concluded that the only modulation that fits with the required BER threshold established in [11] for using HD-FEC $\left(\mathrm{BER}<3.8 \cdot 10^{-3}\right)$ is $64-\mathrm{QAM}$. This transmission is performed at $15 \mathrm{~Gb} / \mathrm{s}$ with a spectrum efficiency of $6 \mathrm{~b} / \mathrm{s} / \mathrm{Hz}$.

The total computational load for the HC-SCM can be obtained by adding the transmitter and the receiver CL presented in Tables $\mathrm{II}$ and $\mathrm{III}$ respectively. This leads to a total CL of $8020 \mathrm{GMult} / \mathrm{s}$, which is an excessive load to be implemented in hardware.

Table IV]presents the throughput, BER and EVM results for the proposed 9/4 OVS-SCM. These results demonstrates that the proposed system is able to transmit up to 256-QAM signals successfully accomplishing the BER threshold using HD-FEC. The maximum spectrum efficiency reached with this proposal is $7.12 \mathrm{~b} / \mathrm{s} / \mathrm{Hz}$ with a throughput of $17.82 \mathrm{~Gb} / \mathrm{s}$. This spectral efficiency is $18 \%$ higher than the one reached by the 64-QAM HC-SCM system.

Tables $\mathrm{V}$ and $\mathrm{VI}$ show the CL of the 9/4 OVS-SCM transmitter and receiver, respectively. The CL of the whole system is $864 \mathrm{GMult} / \mathrm{s}$, that is, $89 \%$ lower than the one obtained from the HC-SCM. 
TABLE V: 9/4 OVS-SCM TRANSMITTER COMPUTATIONAL LOAD

\begin{tabular}{ccccccc}
\hline \hline $\begin{array}{c}\text { TX-9/4 Nyq. } \\
\text { span }\end{array}$ & $\begin{array}{c}C L: T X-9 / 4 N y q . \\
(\text { Gmult/s) }\end{array}$ & $\begin{array}{c}\text { TX-9/4 LPF } \\
\text { order }\end{array}$ & $\begin{array}{c}C L: T X-9 / 4 L P F \\
(\text { Gmult/s })\end{array}$ & $\begin{array}{c}\text { Pre-emph } \\
\text { order }\end{array}$ & $\begin{array}{c}C L: \text { Pre }- \text { emph } \\
(\text { Gmult } / s)\end{array}$ & $\begin{array}{c}\text { Total CL } \\
(\text { Gmult } / s)\end{array}$ \\
\hline 20 & 135.5 & 20 & 33.3 & 2 & 10 & 348 \\
\hline \hline
\end{tabular}

TABLE VI: 9/4 OVS-SCM RECEIVER COMPUTATIONAL LOAD

\begin{tabular}{ccccccc}
\hline \hline $\begin{array}{c}\text { RX-9/4 Nyq. } \\
\text { span }\end{array}$ & $\begin{array}{c}C L: R X-9 / 4 N y q \cdot \\
(\text { Gmult/s) }\end{array}$ & $\begin{array}{c}\text { RX-9/4 LPF } \\
\text { order }\end{array}$ & $\begin{array}{c}C L: R X-9 / 4 L P F \\
(G m u l t / s)\end{array}$ & $\begin{array}{c}\text { RX-9/4 Eq } \\
\text { order }\end{array}$ & $\begin{array}{c}C L: R X-9 / 4 E q \\
(G m u l t / s)\end{array}$ & $\begin{array}{c}\text { Total CL } \\
(G m u l t / s)\end{array}$ \\
\hline 20 & 135.5 & 20 & 33.3 & 40 & 88.8 & 516 \\
\hline \hline
\end{tabular}

Finally, Fig. 11 shows the BER vs. the received optical power (ROP) for both the HC-SCM and the OVS-SCM. It can be seen how the HC-SCM with a 64-QAM modulation order can only work under the HD-FEC threshold for a ROP of $0 \mathrm{dBm}$ or higher. A similar performance is achieved with the OVS-SCM when a 256-QAM modulation order is employed. For lower QAM constellation orders the OVS-SCM can work under the FEC threshold with lower ROP, for example, the crossing with the threshold is lower than $-5 \mathrm{dBm}$ for 128-QAM, whereas for this ROP the 64-QAM signal is under a BER of $10^{-4}$.

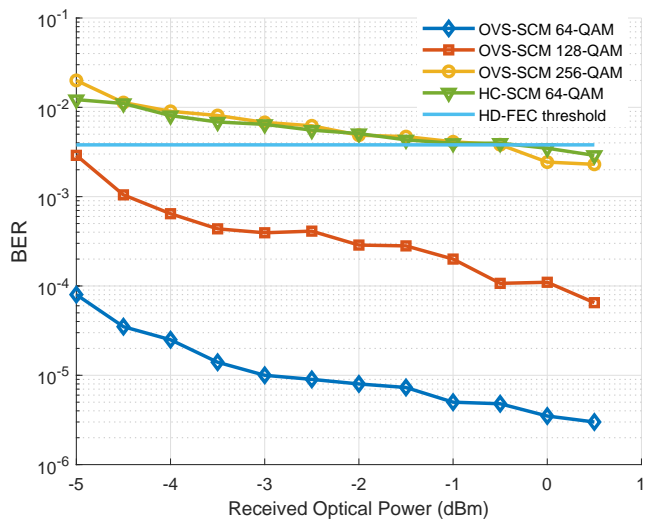

Fig. 11: Dependence of BER on received optical power for HC-SCM 64-QAM, OVS-SCM 64, 128 and 256-QAM. The HD-FEC limit of $\mathrm{BER}=3.8 \cdot 10^{-3}$ is included.

\section{CONCLUSION}

An affordable 64/128/256-QAM for high data-rate transmission over short-reach IM/DD optical links is proposed and experimentally validated using off-the-shell components. This proposal is compared to the HC-SCM system. The proposed system oversamples data with a rational factor of 9/4, which allows the relaxation of the Nyquist filter requirements. Thanks to this fact, the system needs a CL $89 \%$ lower than that of the HC-SCM approach, reaching an spectral efficiency $18 \%$ higher. It was experimentally proved that this system accomplishes the HD-FEC BER threshold, reaching a maximum throughput of $17.8 \mathrm{~Gb} / \mathrm{s}$ for 256-QAM using a bandwidth of $2.5 \mathrm{GHz}$.

\section{ACKNOWLEDGMENT}

This work is funded by the Spanish Ministerio de Economía y Competitividad and FEDER under the grants TEC2015-70858-C2-2-R and RTI2018-101658-B-I00.

\section{REFERENCES}

[1] E. Mohsen, "Designing Nx100G Applications with Heterogeneous 3D FPGAs," Xilinx White Paper, no. 422, pp. 1-9, 2013.

[2] K. Zhong, X. Zhou, J. Huo, C. Yu, C. Lu, and A. P. T. Lau, "Digital Signal Processing for Short-Reach Optical Communications: A Review of Current Technologies and Future Trends," Journal of Lightwave Technology, vol. 36, no. 2, pp. 377-400, 2018.

[3] "ITU-T PON transmission technologies above $10 \mathrm{Gbit} / \mathrm{s}$ per wavelength," Series G, suplement 64, 2018.

[4] J. S. Bruno, V. Almenar, J. Valls, and J. L. Corral, "Real-time 20.37 Gb/s optical OFDM receiver for PON IM/DD systems," Optics Express, vol. 26, no. 15, pp. 18817-18831, 2018.

[5] M. Chen, X. Xiao, Z. R. Huang, J. Yu, F. Li, Q. Chen, and L. Chen, "Experimental Demonstration of an IFFT/FFT Size Efficient DFT-Spread OFDM for Short Reach Optical Transmission Systems," Journal of Lightwave Technology, vol. 34, no. 9, pp. 2100-2105, 2016.

[6] S. Amiralizadeh, A. Yekani, and L. A. Rusch, "Discrete Multi-Tone Transmission with Optimized QAM Constellations for Short-Reach Optical Communications," Journal of Lightwave Technology, vol. 34, no. 15 , pp. $3515-3522,2016$.

[7] Jin Tang, Jing He, Danyu Li, Ming Chen, and Lin Chen, "64/128-QAM Half-Cycle Subcarrier Modulation for Short-Reach Optical Communications," IEEE Photonics Technology Letters, vol. 27, no. 3, pp. 284-287, 2014.

[8] J. C. Cartledge and A. S. Karar, " $100 \mathrm{~Gb} / \mathrm{s}$ intensity modulation and direct detection," Journal of Lightwave Technology, vol. 32, no. 16, pp. 2809-2814, 2014.

[9] A. O. Wiberg, B.-E. Olsson, and P. A. Andrekson, "Single Cycle Subcarrier Modulation," 2009 Conference on Optical Fiber Communication, 2013.

[10] A. S. Karar and J. C. Cartledge, "Generation and detection of a 56 $\mathrm{Gb} / \mathrm{s}$ signal using a DML and Half-Cycle 16-QAM nyquist-SCM," IEEE Photonics Technology Letters, vol. 25, no. 8, pp. 757-760, 2013.

[11] I.-T. S. Group, "ITU-T Rec. G.975.1 (02/2004) Forward error correction for high bit-rate DWDM submarine systems," Standard, pp. 1-58, 2005.

[12] K. Zhong, X. Zhou, Y. Gao, Y. Yang, W. Chen, J. Man, L. Zeng, A. P. T. Lau, and C. Lu, "Transmission of $112 \mathrm{Gbit} / \mathrm{s}$ single polarization half-cycle 16QAM Nyquist-SCM with 25Gbps EML and direct detection," European Conference on Optical Communication, ECOC, vol. 2015-November, pp. 1-3, 2015.

[13] M. Oerder and H. Meyr, "Digital Filter and Square Timing Recovery," IEEE Transactions on Communications, vol. 36, no. 5, pp. 605-612, 1988.

[14] F. Harris, Multirate Signal Processing for Communication Systems. Prentice Hall, 2004. 